Introduction/purpose Higher rates of successful revascularization with the least number of passes correlate with improved clinical outcomes in acute stroke endovascular treatment. Different adjunctive technical approaches such as proximal flow arrest using balloon guide catheter (BGC), large bore conventional guide catheter (CGC), or distal large bore catheter (DLBC) with lesional or regional aspiration, are aimed at improving revascularization rates. We present an interim analysis of adjunctive techniques and angiographic outcomes from the STRATIS Registry.

Materials and methods The STRATIS registry is a prospective, multicenter study of patients with large vessel occlusion (LVO) treated with the Solitaire Stentriever $\leq 8$ hours of symptoms onset. Technical approaches were grouped based on the first technique implemented: BGC; CGC; and DLBC. Posterior circulation target vessel occlusion and subjects with combined BGC and DLBC approach were excluded. A Core Lab extrapolated the techniques from the procedural reports. Baseline variables were compared between the three groups. The main angiographic and technical outcomes were: 1) First pass effect (FPE) defined as successful recanalization of $\geq$ TICI $2 b$, 2) True FPE defined as TICI 3 after first pass with Solitaire; 3) Number of passes among the cohorts.

Results 413 anterior circulation subjects were included in this interim analysis. The initial technical approach was $60 \%$ BGC, $30 \%$ DLBC, and 10\% CGC. The groups were well balanced in reference to baseline and demographic factors. The rates of FPE were: $62 \%, 51 \%$, and $45 \%(\mathrm{P}=0.0336)$, while the true FPE rates were: $44 \%$ vs. $37 \%$ vs. $28 \%(\mathrm{P}=0.0996)$ with BGC, DLBC, and CGC, respectively. The mean number of passes were: $1.7 \pm 1.09,2.1 \pm 1.42$, and $2.2 \pm 1.76$ $(\mathrm{P}=0.0085)$, with BGC, DLBC, and CGC, respectively. The rates of successful recanalization of $\geq \mathrm{TICI} 2 \mathrm{~b}$ after all passes were 91.9\% BGC, 88.8\% DLBC, and 87.5\% CGC $(\mathrm{P}=0.4945)$.

Conclusion The STRATIS registry interim analysis demonstrated a higher use of BGC as first approach (60\%) compared to previous reports. Consistent with published data, BGC is associated with higher rates of successful revascularization and a trend toward higher rates of complete revascularization from the first pass. Moreover, a lower number of passes is associated with BGC use compared to CGC and DLBC. DLBC with lesional and regional aspiration appears to be superior to CGC only. These results are preliminary, and further analysis with final planned sample size and correlation with central blinded core lab imaging data will provide further evidence on technical and angiographic outcomes with different adjunctive approaches.

Disclosures O. Zaidat: 2; C; Medtronic Neurovascular. D. Liebeskind: 1; C; NIH-NINDS. 2; C; Medtronic Neurovascular, Stryker. R. Jahan: 1; C; Medtronic Neurovascular. 2; C; Medtronic Neurovascular. M. Froehler: 2; C; Medtronic Neurovascular. 6; C; Site PI (Large, Liberty, SCENT, Feat, Barrel, Atlas, Rhapsody, Positive, Sep 3D) payment to institution. M. Aziz-Sultan: 2; C; Medtronic Neurovascular. 6; C; Expert Witness - BMC. R. Klucznik: 3; C; Medtronic Neurovascular. J. Saver: 2; C; Medtronic Neurovascular, Stryker, Neuravia, Cognition Medical, Boehringer Ingelheim (prevention only). D. Yavagal: 2; C; Medtronic Neurovascular. 6; C; ESCAPE trial DSCMB member. N. Mueller-Kronast: 2; C; Medtronic Neurovascular.
$0-006$

CORRELATION OF CT AND MRI CLOT CHARACTERISTICS WITH TICI 3 REPERFUSION USING STENT RETRIEVERS IN ACUTE STROKE INTERVENTION

${ }^{1}$ J Wong, ${ }^{2} \mathrm{M}$ Mlynash, ${ }^{3} \mathrm{~N}$ Telischak, ${ }^{1} \mathrm{~A}$ Moraff, ${ }^{3} \mathrm{H}$ Do, ${ }^{1} \mathrm{R}$ Dodd, ${ }^{3} \mathrm{~J}$ Heit, ${ }^{3} \mathrm{M}$ Marks. ${ }^{1}$ Neurosurgery, Stanford, Stanford, $C A ;{ }^{2}$ Neurology, Stanford, Stanford, $C A ;{ }^{3}$ Radiology, Stanford, Stanford, CA

\subsection{6/neurintsurg-2016-012589.6}

Introduction Reperfusion to TICI $2 \mathrm{~B} / 3$ with stent retrievers has significantly improved when compared with intravenous tPA in several recent randomized controlled trials. However, at least $20 \%$ of patients did not achieve this degree of reperfusion. In prior studies, recanalization rates with tPA and early-generation thrombectomy devices (e.g. MERCI) correlated with erythrocyte-rich clots represented by hyperdense MCA (HDMCA) on CT or blooming artifact (BA) on susceptibility-weighted MRI. We hypothesize that clot characteristics, such as clot length, and the presence of HDMCA or BA, may influence the rate of full (TICI 3) reperfusion and the number of passes to achieve reperfusion with stent retrievers.

Methods We retrospectively identified all patients with anterior circulation strokes treated with stent retrievers between January 2015 and March 2016 from our institutional stroke database. All patients underwent a pre-procedural CT or MRI, and revascularization using combined mechanical and aspiration thrombectomy (Solumbra) technique. Patient demographics, risk factors, stroke presentation data, and endovascular treatment details (equipment, number of thrombectomy passes, final TICI reperfusion score, and complications) were recorded. Recorded clot characteristics included the presence of HDMCA and BA on CT and MRI, clot length and Hounsfield Unit density on CT or clot signal intensity on susceptibility-weighted MRI compared to corresponding contralateral artery. Primary outcomes of full reperfusion (TICI 3) and corresponding number of passes were correlated with clot characteristics. Univariate and multivariate analyzes were performed to identify any significant associations.

Results Sixty-four patients with anterior circulation proximal vessel occlusion were treated using stent retrievers. There were $23(36 \%)$ females with a mean age of $73 \pm 14$ years (Range 28-92 years). Median NIHSS on presentation was 14 (IQR 10-19). Vessel occlusion was localized to the ICA terminus (10 patients, 16\%), M1 (41 patients, 64\%) and M2 (13 patients, 20\%) segments. Intravenous tPA was administered in 44 patients (69\%). TICI $2 \mathrm{~B} / 3$ reperfusion was achieved in 57 patients (89\%) and TICI 3 in 27 (42\%). Among HDMCA patients, TICI 3 rate was $50 \%$ (vs $36 \%$ without HDMCA, $\mathrm{p}=0.71$ ); $47 \%$ with $\mathrm{BA}$ (vs $23 \%$ without, $\mathrm{p}=0.27$ ); and $49 \%$ with HDMCA or BA (vs $29 \%$ without, $\mathrm{p}=0.13$ ). No statistical difference was detected between the TICI 3 score and other clot characteristics (clot length, absolute and relative clot density or signal intensity). TICI 3 was significantly associated with single pass revascularization $(74 \%$ vs $41 \%$, $\mathrm{p}=0.008$ ), and time from access to revascularization (30 vs 54 minutes, $\mathrm{p}=0.004)$. Longer clot length on CT correlated to a greater number of passes (Spearman's rho $=0.7$, $\mathrm{p}=0.001)$ and longer time from access to revascularization (rho $=0.47, \mathrm{p}=0.036$ ).

Conclusion A greater percentage of patients with HMDCA or BA will have full (TICI 3) reperfusion compared to patients without HDMCA or BA, however this study was underpowered to demonstrate these differences were significant. Longer 
clot length is correlated with a greater number of passes and longer time to revascularization.

Disclosures J. Wong: None. M. Mlynash: None. N. Telischak: None. A. Moraff: None. H. Do: None. R. Dodd: None. J. Heit: None. M. Marks: None.

\section{0-007 RECANALIZATION AND OUTCOME COMPARISONS IN ACUTE ISCHEMIC STROKE PATIENTS TREATED WITH MECHANICAL THROMBECTOMY SELECTED BY CT/MR PERFUSION IMAGING VERSUS CT ANGIOGRAPHY}

\begin{abstract}
${ }^{1} \mathrm{~A}$ Honarmand, ${ }^{2} \mathrm{~A}$ Shaibani, ${ }^{2} \mathrm{M}$ Hurley, ${ }^{1} \mathrm{P}$ Golnari, ${ }^{2} \mathrm{M}$ Potts, ${ }^{2} \mathrm{~B}$ Jahromi, ${ }^{2} \mathrm{~S}$ Ansari. ${ }^{1}$ Radiology, Northwestern University Feinberg School of Medicine, Chicago, IL; ${ }^{2}$ Radiology and Neurological Surgery, Northwestern University Feinberg School of Medicine, Chicago, IL
\end{abstract}

\subsection{6/neurintsurg-2016-012589.7}

Purpose Several randomized controlled trials, have demonstrated improved percentages of independent clinical outcomes with computed tomography (CT)/magnetic resonance (MR) perfusion selection in acute ischemic stroke (AIS), albeit with more stringent patient exclusion. Conversely, preprocedure CT angiography (CTA) is gaining increasing credence among neurointerventionalists for documenting emergent large vessel occlusion and as a surrogate for tissue selection via collateral imaging. In this study, we compared the level and times to recanalization as well as clinical outcomes in patients selected for endovascular thrombectomy based on their perfusion versus single phase CTA imaging selection.

Materials and methods We performed a prospective IRB approved multicenter study of consecutive AIS patients presenting $<6$ hours from symptom onset that underwent mechanical thrombectomy. Patients were selected after CTA/ MRA confirmed ICA/MCA M1-M2 occlusion and either CTP/ MRP (favorable mismatch ratio/core infarct volumes) or CT/ CTA (ASPECTS $>6$ and pial collaterals $>2 / 3 \mathrm{MCA}$ ) imaging selection. Patient demographics, baseline NIHSS score, THRIVE score, symptom onset to groin puncture times, and the type of thrombectomy devices utilized for recanalization were studied. Modified Rankin Scale score of 0-2 at 90 days defined good functional outcome. Recanalization was delineated by the neurointerventionalist at the end of the thrombectomy according to modified TICI classification. Ordinal recanalization scores of $0,1,2,3$, and 4 were assigned to TICI classification of $0,1,2 \mathrm{a}, 2 \mathrm{~b}$, and 3 , respectively for further evaluation of recanalization reperfusion level. TICI scores of $2 \mathrm{~b}$ and 3 defined successful recanalization Chi-square, student $\mathrm{t}$ test, Mann-Whitney $\mathrm{U}$, and Kruskal-Wallis tests were utilized for statistical analysis as appropriate.

Results Seventy patients $(37 \mathrm{~F} / 33 \mathrm{M}$, mean age $\pm \mathrm{SD}$ of $64.43 \pm 15.08$ years) were recruited into the study. Based on preprocedure imaging, consecutive patients were selected for endovascular thrombectomy either by CTA $(n=35)$ or perfusion studies $(n=35)$. Both groups were comparable in terms of demographics, NIHSS score, THRIVE score, and thrombectomy devices utilized for recanalization. Symptom onset to groin puncture time was significantly lower in the CTA group (mean $=138.50 \mathrm{~min}$ in CTA VS. $224.25 \mathrm{~min}$ in perfusion groups, $\mathrm{P}=0.012$ ). Recanalization scores were not significantly associated with the type of thrombectomy devices $(\mathrm{P}=0.782)$. Although recanalization scores were significantly higher in patients selected by CTA compared with perfusion imaging $(\mathrm{P}=0.020)$, successful recanalization (TICI $2 \mathrm{~b} / 3$ ) was not significantly different $(\mathrm{P}=0.155)$. There was a nonstatistically significant trend for good functional outcome in
CTA group $(\mathrm{P}=0.07)$. Good functional outcome was associated significantly with successful recanalization $(\mathrm{P}<0.005)$.

Conclusions Mechanical thrombectomy patients selected by CTA or perfusion imaging demonstrated no difference in successful recanalization rate, but higher level of recanalization/ reperfusion and a trend of improved outcomes with CTA selection that may be attributed to improved treatment times. Disclosures A. Honarmand: None. A. Shaibani: None. M. Hurley: None. P. Golnari: None. M. Potts: None. B. Jahromi: None. S. Ansari: None.

\section{0-008 FUNCTIONAL AS OPPOSED TO ANATOMICAL CHARACTERIZATION OF THE MIDDLE CEREBRAL ARTERY "M2" DIVISIONS CAN EXPAND THE CATEGORY OF LARGE VESSEL OCCLUSIONS AMENABLE FOR STROKE INTERVENTIONS.}

1A Rai, ${ }^{1}$ A Tarabishy, ${ }^{2} \mathrm{P}$ Link, ${ }^{1} \mathrm{~S}$ Boo, ${ }^{1} \mathrm{~N}$ Lucke-Wold, ${ }^{1} \mathrm{~J}$ Domico, ${ }^{1} \mathrm{~J}$ Carpenter ${ }^{1}$ Interventional Neuroradiology, West Virginia University, Morgantown, WV; ${ }^{2}$ Stryker Neurovascular, Fremont, CA

\subsection{6/neurintsurg-2016-012589.8}

Background M2 occlusions have typically been excluded from endovascular stroke trials. However variations in the size of the MCA branches and the area of brain they supply runs the risk of excluding a dominant trunk supplying a large part of the brain and hence resulting in a significant ischemic injury. Perfusion imaging allows a more functional depiction of the vascular territory and maybe more relevant for endovascular selection.

Objective To determine the rate of M2 occlusions amongst all hospital discharges for acute ischemic stroke (AIS) and to functionally classify occlusion severity based on baseline perfusion imaging and final infarct volume. To secondarily extrapolate this rate to the national inpatient sample (NIS) database for estimating the annual burden of M2 occlusions that may benefit from endovascular therapy.

Methodology All hospital discharges for AIS (ICD-9 codes 433.xx, 434.xx 435.xx) over a 3 year period from a large rural hospital system were screened for an M2 occlusion based on admission imaging. These were classified into the superior or inferior trunk based on anatomy and dominant or non-dominant division based on size. The occlusion severity was graded on time-to-peak (TTP) perfusion imaging as a proportion of the entire MCA. Infarct volume on follow up imaging represented the final ischemic injury. The results were extrapolated to the NIS-database.

Results Out of 2757 AIS hospital discharges 118 (4.3\%) patients were identified with an M2 occlusion. $71(60.2 \%)$ of these patients presented within 6 hours and 47 (39.8\%) after 6 hours of last seen normal (LSN). Baseline perfusion (TTP) and follow-up imaging was available for 75 patients. The superior trunk (ST) was involved in $36(48 \%)$ and the inferior trunk (IT) in 39 (52\%) patients. In 27 (75\%) patients with ST involvement, it was the dominant division versus 36 $(92.3 \%)$ patients with IT occlusion had it as the dominant division ( $\mathrm{p}=0.037$ ). Overall a dominant division was occluded in 63 (84\%) patients and a non-dominant in 12 (16\%) patients. In $47(74.6 \%)$ patients with a dominant branch occlusion (ST or IT) the TTP abnormality was $>1 / 3^{\text {rd }}$ of the MCA distribution versus $5(41.7 \%)$ patients with a non-dominant branch occlusion that had $>1 / 3^{\text {rd }}$ MCA involvement $(\mathrm{p}=0.02)$. Patients with a dominant branch occlusion 